\title{
1 Mutational impact of chronic alcohol use on stem cells in cirrhotic liver
}

2 Myrthe Jager ${ }^{1}$

3 Ewart Kuijk ${ }^{1}$

$4 \quad$ Ruby Lieshout ${ }^{2}$

5 Mauro D. Locati ${ }^{1}$

$6 \quad$ Nicolle Besselink ${ }^{1}$

$7 \quad$ Bastiaan van der Roest ${ }^{1}$

8 Roel Janssen ${ }^{1}$

$9 \quad$ Sander Boymans ${ }^{1}$

10 Jeroen de Jonge ${ }^{2}$

11 Jan N.M. IJzermans ${ }^{2}$

12 Michael Doukas ${ }^{3}$

13 Monique M.A. Verstegen²

14 Ruben van Boxtel ${ }^{1, \uparrow}$

15 Luc J.W. van der Laan ${ }^{2}$

16 Edwin Cuppen ${ }^{1, \#}$

17

$18{ }^{1}$ Center for Molecular Medicine and Oncode Institute, University Medical Center Utrecht, Utrecht

19 University, Heidelberglaan 100, 3584 CX Utrecht, The Netherlands

$20{ }^{2}$ Department of Surgery, Erasmus MC - University Medical Center, Wytemaweg 80, 3015 CN

21 Rotterdam, The Netherlands

$22{ }^{3}$ Department of Pathology, Erasmus MC - University Medical Center, Wytemaweg 80, 3015 CN

23 Rotterdam, The Netherlands

$24{ }^{\dagger}$ Present address: Oncode Institute and Princess Máxima Center for Pediatric Oncology, 3584 CT

25 Utrecht, The Netherlands

$26 \quad$ \#Corresponding author: ecuppen@umcutrecht.nl 


\section{ABSTRACT}

28 Excessive alcohol consumption increases the risk of developing liver cancer, but the mechanism through which alcohol drives carcinogenesis is as yet unknown. Here, we determined the mutational consequences of chronic alcohol use on the genome of human liver stem cells prior to cancer development. No change in base substitution rate or spectrum could be detected. Analysis of the trunk mutations in an alcohol-related liver tumor by multi-site whole-genome sequencing confirms the absence of specific alcohol-induced mutational signatures driving the development of liver cancer. However, we did identify an enrichment of nonsynonymous base substitutions in cancer genes in stem cells of the cirrhotic livers, such as recurrent nonsense mutations in PTPRK that disturb Epidermal Growth Factor (EGF)signaling. Our results thus suggest that chronic alcohol use does not contribute to carcinogenesis through altered mutagenicity, but instead induces microenvironment changes which provide a 'fertile ground' for selection of cells with oncogenic mutations.

INTRODUCTION

Alcohol consumption is an important risk factor for the development of various cancer types, including hepatocellular carcinoma (HCC), and causes an estimated 400,000 cancer-related deaths worldwide each year ${ }^{1-3}$. In spite of the clear link between alcohol intake and tumorigenesis, the underlying mechanism remains debated and mainly revolves around two hypotheses. The first hypothesis suggests that alcohol consumption may contribute to the

47 development of cancer through an increased mutation accumulation in the genome ${ }^{4}$.

48 Consistently, the first metabolite of ethanol, acetaldehyde, is highly carcinogenic ${ }^{5-7}$ and can also contribute to the formation of mutagenic reactive oxygen species $(\mathrm{ROS})^{8-11}$. Analysis of a

50 large number of tumor exomes and genomes showed that alcohol intake is associated with an 51 increased mutation load and different mutational characteristics ${ }^{12-15}$. The second hypothesis 
suggests that an alcohol-induced change of microenvironment is an essential driver for tumorigenesis by providing a fertile ground for cells with oncogenic mutations ${ }^{16-18}$. Indeed, development of HCC is preceded by chronic inflammation and cirrhosis in about $80 \%$ of patients and this cell-extrinsic damage appears a prerequisite for the formation of the majority of liver cancers ${ }^{18-20}$. Additionally, Hepatitis C Virus (HCV)-induced cirrhotic livers show an increase in the number and size of clonal patches with mutations in genes that are frequently mutated in $\mathrm{HCC}^{21}$. Alcohol use itself has been associated with an increased number of cancerstem-cell-like epithelial cell adhesion molecule (EpCAM)-positive cells in the cirrhotic liver ${ }^{22}$, which may be driven by epithelial to mesenchymal transition through activation of the Wnt pathway $^{23}$, confirming that cellular composition changes can be induced by alcohol use. Yet, it is still uncertain whether an altered cellular environment is sufficient to drive the development of cancer, or whether an increase in the mutation load is also required. The here mentioned hypotheses are thus not mutually exclusive.

We have demonstrated previously that mutations accumulate linearly with age in liver adult stem cells (ASCs) of healthy individuals, without controlling for lifestyle ${ }^{24,25}$. Stem cells are believed to be an important cell-of-origin for several cancer types, including liver cancer $^{18,26-28}$, although liver cancer can also originate in differentiated cells ${ }^{29-31}$. Here, we studied the accumulation of mutations in ASCs from non-cancerous, cirrhotic livers of patients with a history of chronic alcohol use and compared these to the mutational patterns of healthy liver donors and to mutations that accumulated in the most recent common ancestor (MRCA) cell of an alcohol-related HCC.

\section{RESULTS}


We sequenced the genomes of eight independent clonal organoid cultures derived from

77

78

biopsies of five non-cancerous, cirrhotic livers from patients with a known history of chronic alcohol intake who were undergoing a liver transplantation (further referred to as 'alcoholic livers'; Supplementary Table 1). To gain insight into the mutational consequences of chronic alcohol consumption, the somatic mutation catalogs of alcoholic livers were compared to those obtained previously from whole genome sequencing (WGS) data of five healthy liver donors (further referred to as 'healthy livers') ${ }^{24}$. To increase the number of healthy liver donors and to obtain age-matched healthy controls, five clonal liver organoid cultures derived from four additional healthy liver donors with ages ranging from 24 to 68 years were included in the analyses (Supplementary Table 1).

In total, we identified 42,093 base substitutions, 1,931 indels, and 5 copy number alterations (CNAs) (Fig. 1; Supplementary Fig. 1; Supplementary Table 1). Consistent with previous observations ${ }^{24}$, there is a positive relationship between somatic base substitutions and age in healthy liver ASCs (two-tailed $t$-test, linear mixed model, $P<0.05$; Fig. 1a). Healthy liver ASCs acquired 39.4 (95\% confidence interval (95\% CI): 30.5 - 48.3) somatic base substitutions each year. The mutation load in alcoholic liver ASCs (Fig. 1a) was similar to, and within the $95 \%$ CI of the slope estimate of, age-matched healthy liver ASCs. Alcohol consumption did not affect the number of tandem base substitutions acquired in the genomes of liver ASCs either (Supplementary Fig. 1a). Furthermore, indels also accumulated with age at a comparable rate in healthy and alcoholic liver ASCs (two-tailed $t$-test, linear mixed model, $P<0.05$; Supplementary Fig. 1b). Finally, a minority of the healthy liver ASCs and none of the assessed alcoholic liver ASCs acquired a CNA, although few CNAs were detected (Supplementary Table 1$)^{24}$. At the chromosomal level we observed trisomy 22 in a healthy liver ASC from a 68-year-old healthy female donor and chromosome Y gain in an alcoholic liver ASC from a 67-year-old male donor (Supplementary Fig. 1c). This suggests that 
aneuploidies may occur in liver ASCs of older individuals, but this seems to be unrelated to accumulation in liver ASCs prior to oncogenesis.

\section{Mutation type similar in alcoholic liver}

It has been shown that genome-wide patterns of base substitutions reflect past activity of mutational processes in cells ${ }^{32}$. Previously, alcohol consumption was reported to be associated with a modest increase of Catalogue Of Somatic Mutations In Cancer (COSMIC) signature 16 mutations, which is characterized by $\mathrm{T}: \mathrm{A}>\mathrm{C}: \mathrm{G}$ mutations ${ }^{32}$, in esophageal and liver cancer ${ }^{12-}$ 15. To identify if excessive alcohol consumption changed the mutational profiles in noncancerous liver ASCs, we performed in-depth mutational analyses. The mutational profiles of healthy liver ASCs were characterized by a high contribution of $\mathrm{C}: \mathrm{G}>\mathrm{A}: \mathrm{T}, \mathrm{C}: \mathrm{G}>\mathrm{T}: \mathrm{A}$, and T:A $>$ C:G mutations (Fig. 1b-c; Supplementary Fig. 2). The mutational profiles of alcoholic liver ASCs were highly similar to the mutational profiles of healthy liver ASCs (cosine similarity $=0.99)$, indicating that chronic alcohol use does not alter the mutational processes in liver ASCs.

To determine whether chronic alcohol use changed the contribution of the known COSMIC mutational signatures ${ }^{32-34}$, we calculated the contribution of these signatures to the mutational profiles of all ASCs and, subsequently, performed a bootstrap resampling method to identify potential significant differences between healthy and alcoholic liver ASCs, similar as described in Zou et al. ${ }^{35}$. COSMIC signatures 5 and 40 could explain the majority of the accumulated base substitutions in both healthy and alcoholic liver (Supplementary Fig. 3). 
liver ASCs and healthy liver ASCs (bootstrap resampling method, see Methods;

A possible explanation for the absence of a correlation between alcohol consumption and mutational patterns is that the cells that we have sequenced are too early in the precancerous state. Therefore, we also sequenced five biopsies across a $13 \mathrm{~cm} \mathrm{HCC}$ of a 60 -year-old male donor with a history of chronic alcohol use and identified mutations that were shared by all biopsies (Fig. 2a; Supplementary Fig. 4). This approach allowed for the identification of all mutations in the MRCA and thus provided insight into the mutational process that had been active prior to tumor formation and in the early to intermediate stages of tumor development (Fig. 2a). As a control sample, we sequenced a non-tumorous biopsy adjacent to the tumor, to identify and exclude germline mutations. In total, we identified 19,200 unique somatic base substitutions across all five HCC biopsies (Supplementary Table 2; Supplementary Fig. 4b). the MRCA of these biopsies accumulated 7,203 base substitutions (Supplementary Table 2;

139 Supplementary Fig. 4b). The mutational profile of the trunk mutations in the HCC was highly 140 similar to healthy and alcoholic liver ASCs (Fig. 2b; cosine similarity $=0.97$ and 0.98 , respectively). These results were in line with our initial observations in ASCs that alcohol itself does not introduce specific mutations in the genome of liver cells. The high mutation load suggests that the MRCA already evolved significantly from the cell-of-origin (Fig. 2a) and that

144 a clonal sweep occurred after a substantial amount of mutations already accumulated.

145 Consistently, the MRCA already acquired two CNAs (Supplementary Table 3) and several chromosomal aneuploidies (Fig. 2c; Supplementary Fig. 4c).

\section{Cancer driver mutations}


149 Previously, alcohol intake has been shown to accelerate the expansion of clones with cancer

150 driver mutations in the esophagus ${ }^{36}$. To identify whether chronic alcohol consumption induces

151 similar changes in the selection of liver cells, we analyzed the genomic distribution of the

152 acquired base substitutions. If chronic alcohol use would affect cellular selection, the frequency

153 of somatic mutations in active functional genomic elements would differ between alcoholic and healthy liver. Base substitutions were, however, depleted to a similar extend in regions such as genes and enhancers in healthy liver ASCs and alcoholic liver ASCs (Fig. 3a). Furthermore, unlike previous observations ${ }^{37}$, we did not observe an enrichment of base substitutions in $\mathrm{H} 3 \mathrm{~K} 36 \mathrm{me} 3$ regions, associated with active transcription ${ }^{38,39}$, in alcoholic liver

158 ASCs in comparison to healthy liver ASCs (Supplementary Fig. 5). The normalized ratio of nonsynonymous to synonymous base substitutions $(\mathrm{d} N / \mathrm{d} S)$ was also $\sim 1$ in all assessed cell types (Fig. 3b). Taken together, these results suggest that there is no general change in selection against more deleterious base substitutions.

However, we observed a small enrichment of potential driver mutations in alcoholic liver ASCs (Fig. 3c; Table 1), although the number of mutations was low. Only one in three healthy liver ASCs acquired a nonsynonymous base substitution in a COSMIC cancer census gene. In alcoholic liver ASCs, on the other hand, we observed a total of seven nonsynonymous base substitutions in these cancer genes across eight ASCs. Two alcoholic liver ASCs even acquired multiple nonsynonymous hits in cancer genes (Fig. 3c; Table 1), while only an estimated four nonsynonymous base substitutions in cancer genes is sufficient to drive the 169 development of liver cancer ${ }^{16}$. Consistent with this idea, we identified four nonsynonymous base substitutions in cancer genes in the MRCA of the HCC (Fig. 3c; Table 1). The modest

171 increase in nonsynonymous base substitutions in cancer genes observed in alcoholic liver ASCs

172 suggests that alcohol may cause clonal outgrowth of cells with putative oncogenic mutations, 173 similar to alcohol-exposed esophagus ${ }^{36}$ and $\mathrm{HCV}$-induced liver cirrhosis ${ }^{21}$. 
(Table 1; likelihood ratio test, FDR correction; $q=0.02)^{16}$. None of the healthy liver ASCs acquired a nonsynonymous base substitution in PTPRK, nor did we identify nonsynonymous base substitutions in this gene in healthy ASCs from small intestine or colon ${ }^{24}$. RNAsequencing of the organoids revealed that the heterozygous nonsense base substitutions in PTPRK resulted in a significantly reduced expression (Fig. $4 ; P<0.05$, negative binomial test), indicating a gene dosage effect due to nonsense-mediated decay of the mutated allele. PTPRK can modulate EGF-signaling through dephosphorylation of tyrosine residues of the EGFR ${ }^{40}$. Western blot analysis showed that alcoholic $P T P R K^{\mathrm{WT} / *}$ cells had increased pERK levels in the absence of EGF, indicating that the PTPRK mutations indeed disturb EGF-signaling (Fig. 4).

\section{DISCUSSION}

188 In this study, we aimed to identify the mutational consequences of chronic alcohol intake in cirrhotic livers, prior to the development of liver cancer. In contrast to previous studies ${ }^{12-15,37}$, we did not observe specific mutational signatures associated with alcohol consumption in stem cells from non-cancerous cirrhotic livers. This observation can indicate that chronic alcohol use may only impact directly on mutation accumulation after tumor initiation or it may reflect very effective negative selection of cells with DNA damage. Alternatively, it should be noted that tissue-specific liver ASCs may not be the direct cell-of-origin for HCC. Nevertheless, as the cancer-initiating cells are exposed to the same mutagenic damage as the liver ASCs (and show the same mutational signatures), our results suggest that alcohol-induced cancer risk is not caused by altered mutagenesis. 

causes apoptosis and necrosis of various cells in the liver, such as the hepatocytes, leading to

202 liver inflammation ${ }^{41}$. As a consequence, tissue-specific ASCs, which are normally quiescent, will proliferate to aid in the regeneration of the damaged liver ${ }^{42}$. Oncogenic mutations that have accumulated randomly in ASCs through normal mutational processes could provide a proliferative advantage under these inflamed conditions at the expense of 'normal' ASCs that do not carry such mutations, while there is too little proliferation under normal conditions for ASCs with oncogenic mutations to outcompete normal ASCs. In the damaged liver, the enhanced proliferation of such potential cancer progenitor cells could subsequently result in an increasing number of mutations that drive tumorigenesis further, as long as inflammation persists.

An illustrative example of this potentially altered selection process in the current study 212 is the significant enrichment of nonsense base substitutions in EGFR phosphatase PTPRK in 213 alcoholic liver ASCs. Reduced expression of PTPRK has been reported to cause enhanced 214 EGF-signaling and ultimately increases cellular proliferation ${ }^{40,43,44}$. Single-nucleotide 215 polymorphisms in EGF that prolong the half-life of EGF increase the risk of developing HCC through continued EGF-signaling $17,45,46$ and $22 \%$ of HCCs carry mutations in genes in the EGF 217 pathway ${ }^{47}$. Reduced EGF-signaling, on the other hand, significantly decreases tumor formation 218 in cirrhotic livers from rats ${ }^{48}$. These observations underscore the importance of (disturbed) 219 EGF-signaling in HCC development. Nonsense mutations in PTPRK may thus contribute to the development of HCC by changing EGF-signaling as well, although further research should

221 be conducted to identify the significance of our findings in liver cancer. 

carcinogenesis. Inflammatory diseases, such as inflammatory bowel disease and pancreatitis, increase the risk of developing cancer in various tissues ${ }^{49}$. However, it was believed that this increased risk was at least partially due to a direct induction of mutations in the genome ${ }^{50}$. The results presented here indicate that alteration of selective mechanisms induced by inflammation could be more directly involved in the development of cancer. For liver, reversal of the inflammatory phenotype that precedes cancer might aid in reducing cancer risk in patients with cirrhotic liver disease due to chronic alcohol use.

\section{METHODS}

\section{Human tissue material}

233 All human tissue biopsies were obtained in the Erasmus MC - University Medical Center Rotterdam. Liver biopsies from healthy liver donors and patients with alcoholic cirrhosis were obtained during liver transplantation procedures. All patients were negative for viral infection and metabolic diseases. The biopsies were collected in cold organ preservation fluid (Belzer UW Cold Storage Solution, Bridge to Life, London, UK) and transported and stored at $4^{\circ} \mathrm{C}$ until use. The liver and tumor biopsies from the hepatocellular carcinoma patient were collected from a resected specimen and stored at $-80^{\circ} \mathrm{C}$ until use. The acquisition of these liver and tumor biopsies for research purposes was approved by the Medical Ethical Committee of the Erasmus Medical Center (MEC-2014-060 and MEC-2013-143). Informed consent was provided by all patients involved. 


\section{Generation of clonal liver organoid cultures from human liver biopsies}

249 Organoid cultures from healthy and alcoholic liver tissue material were derived as previously 250 described ${ }^{51,52}$. After 2 - 3 days, organoids started to appear in the BME. The cultures were maintained for approximately 2 weeks after isolation, to enrich for ASCs. Subsequently, clonal organoid cultures were generated from these organoid cultures as described previously ${ }^{25}$. The organoid cultures were expanded until there was material for WGS.

\section{Whole-genome sequencing and read alignment}

DNA was isolated from all organoid cultures, blood samples, and tissue biopsies using the

Qiasymphony (Qiagen). Whole-genome sequencing libraries were generated from $200 \mathrm{ng}$ of genomic DNA according to standard Illumina protocols. The organoid cultures and control samples were sequenced paired-end $(2 \times 150 \mathrm{bp})$ to a depth of at least $30 \mathrm{X}$ coverage using the Illumina HiSeq Xten. The HCC biopsies were sequenced paired-end ( 2 x 150bp) to a depth of at least $60 \mathrm{X}$ coverage using the Illumina HiSeq Xten. A $60 \mathrm{X}$ depth was required to identify the somatic base substitutions in the tumor cells, as the biopsies contain $\sim 50 \%$ healthy cells reference genome GRCh37 using the Burrows-Wheeler Aligner (BWA) tool v0.7.5a $\mathrm{a}^{53}$ (settings -t, 4, -c, 100, -M).

\section{Copy number alteration calling and filtering}

269 For the healthy and alcoholic samples without HCC, CNA catalogs were obtained and filtered 270 by using FreeC v2. $7^{25,54}$. Calls were excluded if the mapping quality of the split reads was 0 on 271 either sides of the split read. BED-file of blacklist positions is available upon request. For the 
HCC biopsies, structural variants were called using Manta v.1.1.0 ${ }^{55}$ with standard settings. We

273

274

275 only considered structural variations of at least 150 base pairs in autosomal the genome with a manta filter 'PASS'. Subsequently, the mutation catalogs of all five biopsies were intersected with a window of $500 \mathrm{bp}$ to obtain the trunk CNAs using bedtools ${ }^{56}$.

All CNA calls were inspected manually in the Integrative Genomics Viewer (IGV) to exclude false-positives with no change in read-depth. The breakpoints were identified manually in IGV. Finally, the number of genes within the deletions was obtained from http://genome.ucsc.edu/.

\section{Genome-wide copy number profiles}

Genome-wide copy number profiles of the ASCs were estimated by using the output of the FreeC calls obtained in section 'Copy number alteration calling and filtering' prior to filtering. Subsequently, we calculated the mean copy number across 500,000 bp bins. Copy number of $\geq 2.8$ was considered a gain and copy number of $\leq 1.2$ a loss. Genome-wide copy number profiles of the HCC biopsies and the adjacent liver biopsy were obtained in a similar manner.

\section{Base substitution calling and filtering}

For the organoid cultures, base substitution catalogs were obtained by filtering GATK v3.4$46^{57}$ variant calls as previously described ${ }^{25}$, with additional removal of variants with a samplespecific genotype quality $<10$ in the control sample, and positions with a sample-specific genotype quality $<99$ in the organoid clone sample. The callable regions, used to define regions with high confidence base substitutions, were obtained by using the GATK CallableLoci tool v3.4.46 $6^{58}$ as previously described ${ }^{25}$. BED-file of blacklist positions is available upon request. All organoids showed a peak at a base substitution VAF of 0.5 , confirming that the organoid samples are clonal (Supplementary Fig. 7). Publicly available variant call format (VCF) files 

healthy and alcoholic liver ASCs.

For the HCC biopsies, base substitutions were called by using Strelka v1.0.14 with settings 'SkipDepthFilters $=0$ ', 'maxInputDepth $=250$ ', 'depthFilterMultiple $=3.0$ ', 'snvMaxFilteredBasecallFrac $=0.4$ ', 'snvMaxSpanningDeletionFrac $=0.75$ ', 'indelMaxRefRepeat = 1000', 'indelMaxWindowFilteredBasecallFrac $=0.3^{\prime}$, 'indelMaxIntHpolLength $=14$ ', 'ssnvPrior $=0.000001$ ', 'sindelPrior $=0.000001$ ', 'ssnvNoise $=0.0000005$ ', 'sindelNoise $=0.000001$ ', 'ssnvNoiseStrandBiasFrac $=0.5$ ', 'minTier1Mapq $=$ 20', 'minTier2Mapq $=5$ ', 'ssnvQuality_LowerBound $=10$ ', 'sindelQuality_LowerBound $=$ 10 ', 'isWriteRealignedBam $=0$ ', and 'binSize $=25000000$ '. We only considered variations with a filter 'PASS'. Subsequently, the mutation catalogs of all five biopsies were intersected to obtain the trunk mutations using bedtools ${ }^{56}$. We only considered base substitutions on the autosomal genome that did not overlap with an indel call. Positions that were detected at least 5 times in 1,762 Dutch individuals were removed from these catalogs using the Hartwig Medical Foundation Pool of Normals (HMF-PON) version 2 (available upon request), to exclude Dutch germline variations. Only 138 base substitutions are found in four out of five biopsies, whereas we detect 7,203 base substitutions in all five biopsies (Supplementary Fig. 4), indicating that the majority of the trunk mutations were identified successfully.

To exclude that the observed similarities/differences in base substitution load and type are a consequence of the differences between the filtering pipelines, we also applied the filtering steps of the HCC samples to the base substitutions in the alcohol liver ASCs. We observe no obvious differences in base substitution load or type between the alcoholic liver samples using both filtering pipelines (Supplementary Fig. 8). 


\section{Tumor adjusted allele frequencies}

323 The VAFs of the shared base substitutions (the trunk mutations) were calculated for each

324 biopsy. Subsequently, we calculated the tumor-adjusted variant allele frequency (TAF) per 325 biopsy, in which the VAF is divided by the tumor-fraction. Chromosome 1 and chromosome 8 were excluded from these analyses, as these chromosomes deviate from a copy number of two in the majority of the biopsies (Supplementary Fig. 4). Most biopsies showed a peak around a TAF of 0.5 (Supplementary Fig. 9), confirming that these base substitutions are clonal in each sample and that the biopsies share a recent common ancestor.

\section{Indel calling and filtering}

WGS data of previously published samples was obtained from EGAD00001001900. Indels were called using GATK v3.4-46 ${ }^{57}$. We only considered indels that were callable/surveyed on false positive calls, indels with a GATK quality score $<250$ and/or with a mapping quality $<$ 60 were excluded. Additionally, only indels with a coverage of at least 20X and a GATK sample-specific quality score of at least 99 in both control and organoid clone sample were considered. Subsequently, variants with a cosmic and/or a dbSNP id (dbSNP v137.b3730) and indels that were found in three unrelated control samples (BED-file available upon request) were excluded. To obtain a catalog of somatic indels, we excluded indels with any evidence in

341 the reference sample, and that were located within 100 base pairs of an indel that was called in

342 the reference sample. Finally, we only considered variants with a VAF of $\geq 0.3$ in the organoid 343 clone sample. 
The number of base substitutions in the genomes of liver ASCs was obtained from the VCF

347 files and extrapolated to the non-N autosomal genome $(2,682,655,440 \mathrm{bp})$ of GRCh37 using

348 the callable/surveyed genome size obtained in section 'Base substitution calling and filtering'.

349 To identify whether the number of somatic base substitutions acquired in the genomes of liver

350 ASCs are correlated with the age of the donor, we fitted a linear mixed-effects regression model with the donor as a random effect in this model using the nlme $\mathrm{R}$ package, as described previously ${ }^{24}$. Two-tailed $t$-tests were performed to determine whether the correlation between age and number of mutations was significant. The accumulation of base substitutions did not correlate significantly with age in the alcoholic liver ASCs ( $\sim 38.6$ somatic base substitutions per year; 95\% CI: -51.8 - 128.9; two-tailed $t$-test, linear mixed model, non-significant), most likely due to the fact that the age-range is much smaller in these donors. Therefore, we obtained the $95 \%$ CI of the healthy liver ASCs from the output of the linear mixed-effects regression model and determined whether the number of somatic base substitutions acquired in the genomes of the alcoholic liver ASCs are within this 95\% CI.

To identify tandem base substitutions, we extracted base substitutions that were called on two consecutive bases in the GRCh37 human reference genome from the VCF files. Similar to single base substitutions, we extrapolated this number to the non-N autosomal genome and determined whether the number of tandem base substitutions was correlated with the age of the donor using a linear mixed effects regression model. As the number of tandem base substitutions did not significantly correlate with age in the alcoholic liver ASCs $(\sim 0.04$ tandem base substitutions per year; 95\% CI: -1.13 - 1.21; two-tailed $t$-test, linear mixed model, nonsignificant), we determined whether these tandem base substitution numbers are within the $95 \% \mathrm{CI}$ of the healthy liver ASCs. 
a linear mixed effects regression model with the donor as random effect, we assessed whether

372 the number of indels was correlated with the age of the donor. Two-tailed $t$-test were performed

373 to determine whether the correlation was significant for both alcoholic liver ASCs and healthy

374 liver ASCs.

\section{Mutational pattern analysis}

377 Mutation types of the base substitutions were extracted from the VCF files and the mutational profiles were generated by retrieving the sequence context of each base substitution. For the healthy and alcoholic liver ASCs, we calculated an 'average' mutational profile. Pairwise cosine similarities of these average mutational profiles and of the mutational profile of the trunk mutations of the HCC were calculated, to identify the similarity between these profiles. We reconstructed the mutational profiles of the average mutational profiles and the trunk mutations using the 60 known SBS signatures (Supplementary Fig. 3;

https://cancer.sanger.ac.uk/cosmic/signatures v3). A bootstrap resampling method similar as

385 described in Zou et al., ${ }^{35}$ was used to generate $120,000(8 \times 15 \times 1,000)$ replicas of the mutational profiles of the healthy and alcoholic liver ASCs. Subsequently, 8 or 15 (for healthy and alcoholic liver ASCs respectively) replicas were randomly selected and an average mutational profile was calculated. This was repeated 10,000 times, to obtain 10,000 average mutational profiles of the replicas for both healthy and alcoholic livers. These average 390 mutational profiles of the replicas were reconstructed using the 60 known SBS mutational 391 signatures and the Euclidean distance to the original signature contribution was calculated for each reconstructed average mutational profile. Next, the distance at which $P=0.01$ was determined for both healthy and alcoholic liver ASCs ( $d_{\text {healthy }=0.01}$ and $d_{\text {alcoholic }=0.01}$, respectively). The Euclidean distance $(d)$ between the original signature contributions of 
healthy and alcoholic liver ASCs was considered significant when $d$ was larger than $d_{\text {healthy }=0.01}$

and $d_{\text {alcoholic }=0.01 \text {. }}$

\section{Genomic distribution of somatic base substitutions}

399 The promoter, enhancer, and open chromatin regions of hg19 were obtained from Ensembl 400 using biomaRt $\mathrm{t}^{59,60}$ and the genic regions of hg19 were loaded from UCSC Known Genes tables 401 as $\mathrm{TxDb}$ object $^{61}$. To determine whether the somatic base substitutions are non-randomly distributed, we tested for enrichment and depletion of base substitutions in these regions with a one-sided Binomial test, corrected for the callable/surveyed regions per sample, similar as described in ${ }^{24}$. For the HCC trunk mutations, the callable regions were obtained by defining callable loci per biopsy using the GATK CallableLoci tool v3.4.46 ${ }^{58}$ (optional parameters 'minBaseQuality 10', 'minMappingQuality 10', 'maxFractionOfReadsWithLowMAPQ 20', and 'minDepth 15'). Subsequently, these files were intersected to obtain the regions that are callable in all biopsies. $96.79 \%$ of the non-N autosomal genome was callable in all six biopsies. Two-sided poisson tests were done to estimate significant differences in depletion/enrichment 410 in all genomic regions between the healthy liver ASCs, the alcoholic liver ASCs, and the trunk mutations of the HCC. Differences were considered significant when $q<0.05$ (BenjaminiHochberg FDR multiple-testing correction). All mutational pattern analyses were performed using the MutationalPatterns R package ${ }^{62}$.

To obtain a generic genome-wide profile of $\mathrm{H} 3 \mathrm{~K} 36 \mathrm{me} 3$, we downloaded and merged 41540 available H3K36me3 ChIP-Seq datasets from UCSC, and determined the median $416 \mathrm{H} 3 \mathrm{~K} 36 \mathrm{me} 3$ values in regions that show H3K36me3 enrichment in at least 2 of the datasets.

417 H3K36me3 peaks were subsequently called using bdgpeakcall function of MACS2 (broad 418 peaks $)^{63}$. The amount of base substitutions that overlap with these peaks was calculated for all 419 base substitutions acquired in liver ASCs. These analyses were repeated for T:A > C:G 
mutations specifically. Wilcoxon-rank tests were performed to estimate significant differences

421

422

423

424

425

426

427

428

429

430

431

432

433

434

435

436

437

438

439

440

441

442

443 in the relative amount of base pair substitutions in $\mathrm{H} 3 \mathrm{~K} 36 \mathrm{me} 3$ regions between alcoholic and healthy liver ASCs. Differences with $P<0.05$ were considered significant.

\section{$\mathrm{d} N / \mathrm{d} S$ and identification of nonsynonymous base substitutions in cancer genes}

$\mathrm{d} N / \mathrm{d} S$ ratios were computed using the $d N d S c v$ R package ${ }^{16}$. The output of the $d N d S c v$ package was used to identify missense, nonsense, and splice site base substitutions in cosmic cancer genes. For this analysis, we considered all 409 'tier 1' cancer genes (genes with sufficient evidence of being a cancer driver). The list of cosmic cancer genes was obtained from

\section{https://cancer.sanger.ac.uk/cosmic/census.}

\section{RNA sequencing}

Organoid cultures of three healthy donors (18-c, 21-b, and 22-a) and three alcoholic organoids, of which 2 with a nonsense base substitution in PTPRK (alc3-a and alc5-a) and one without any base substitutions in PTPRK (alc-3b), were cultured for 1 day either in presence or absence of hEGF in the culture medium. Subsequently, cells were collected in Trizol. Total RNA was isolated using the QiaSymphony SP with the QiaSymphony RNA kit (Qiagen, 931636). mRNA sequencing libraries were generated from 50 ng total RNA using the Illumina Neoprep TruSeq stranded mRNA library prep kit (Illumina, NP-202-1001). RNA libraries were sequenced paired-end $(2 \times 75 \mathrm{bp})$ on the Nextseq500 to $>20$ million reads per sample at the Utrecht Sequencing facility.

RNA sequencing reads were mapped to the human reference genome GRCh37 with STAR v.2.4.2 $\mathrm{a}^{64}$. The BAM-files were indexed using Sambamba v0.5.8 Subsequently, reads were counted using HTSeq-count 0.6.1 and read counts were normalized using DESeq v1.18.0. 
444 DESeq nbinomTest was used to test for differential expression of PTPRK between the 445 organoids with a nonsense PTPRK base substitution and the other organoids.

446

\section{Western blot}

448 Simultaneous to the collection of samples for RNA isolation described above, we also obtained 449 protein samples for western blot in Laemmli buffer. 20 ug of protein was run on a 10\% SDS 450 page gel and blocked for 1 hour using 5\% ELK in TBS-T after transfer to a nitrocellulose 451 membrane. Subsequently, the membrane was incubated overnight with primary antibody 452 (pERK AB50011, abcam; ERK AB17942, abcam; Actin A2066, sigma-aldrich) and for 1 hour 453 at room temperature with secondary antibody. We visualized the proteins with the Amersham 454 ECL Western blotting analysis system (GE Healthcare, RPN2109).

\section{Data availability}

457 The whole-genome sequencing and RNA sequencing data generated during the current study 458 are available at EGA (https://www.ebi.ac.uk/ega/home) under accession number 459 EGAS00001002983. Filtered VCF-files, metadata, BED-files with callable regions, and RNA460 Seq counts generated during the current study are available at Zenodo under DOI 10.5281/zenodo.3295513 (https://doi.org/10.5281/zenodo.3295513). Data analysis scripts used during the current study are available at https://github.com/UMCUGenetics/Liverdisease, 463 https://github.com/UMCUGenetics/IAP and https://github.com/hartwigmedical.

\section{REFERENCES}


(2006).

470

471

472

473

474

475

476

477

478

479

480

481

482

483

484

485

486

487

488

489

490

491

492

493

3. World Health Organization. Global Status Report on Alcohol and Health. (World Health Organization, 2014).

4. Mizumoto, A. et al. Molecular Mechanisms of Acetaldehyde-Mediated Carcinogenesis in Squamous Epithelium. Int. J. Mol. Sci. 18, (2017).

5. Obe, G. \& Ristow, H. Mutagenic, cancerogenic and teratogenic effects of alcohol. Mutat. Res. 65, 229-259 (1979).

6. Helander, A. \& Lindahl-Kiessling, K. Increased frequency of acetaldehyde-induced sister-chromatic exchanges in human lymphocytes treated with an aldehyde dehydrogenase inhibitor. Mutat. Res. Lett. 264, 103-107 (1991).

7. Matsuda, T., Kawanishi, M., Matsui, S., Yagi, T. \& Takebe, H. Specific tandem GG to TT base substitutions induced by acetaldehyde are due to intra-strand crosslinks between adjacent guanine bases. Nucleic Acids Res. 26, 1769-1774 (1998).

8. Tamura, M., Ito, H., Matsui, H. \& Hyodo, I. Acetaldehyde is an oxidative stressor for gastric epithelial cells. J. Clin. Biochem. Nutr. 55, 26-31 (2014).

9. Novitskiy, G., Traore, K., Wang, L., Trush, M. A. \& Mezey, E. Effects of ethanol and acetaldehyde on reactive oxygen species production in rat hepatic stellate cells. Alcohol. Clin. Exp. Res. 30, 1429-1435 (2006).

10. Grollman, A. P. \& Moriya, M. Mutagenesis by 8-oxoguanine: an enemy within. Trends Genet. 9, 246-249 (1993).

11. van Loon, B., Markkanen, E. \& Hübscher, U. Oxygen as a friend and enemy: How to combat the mutational potential of 8-oxo-guanine. DNA Repair 9, 604-616 (2010).

12. Chang, J. et al. Genomic analysis of oesophageal squamous-cell carcinoma identifies alcohol drinking-related mutation signature and genomic alterations. Nat. Commun. 8, 15290 (2017). 
494

495

496

497

498

499

500

501

502

503

504

505

506

507

508

509

510

511

512

513

514

515

516

517

518

13. Schulze, K. et al. Exome sequencing of hepatocellular carcinomas identifies new mutational signatures and potential therapeutic targets. Nat. Genet. 47, 505-511 (2015).

14. Fujimoto, A. et al. Whole-genome mutational landscape and characterization of noncoding and structural mutations in liver cancer. Nat. Genet. 48, 500-509 (2016).

15. Letouzé, E. et al. Mutational signatures reveal the dynamic interplay of risk factors and cellular processes during liver tumorigenesis. Nat. Commun. 8, 1315 (2017).

16. Martincorena, I. et al. Universal Patterns of Selection in Cancer and Somatic Tissues. Cell 171, 1029-1041.e21 (2017).

17. Hernandez-Gea, V., Toffanin, S., Friedman, S. L. \& Llovet, J. M. Role of the Microenvironment in the Pathogenesis and Treatment of Hepatocellular Carcinoma. Gastroenterology 144, 512-527 (2013).

18. Zhu, L. et al. Multi-organ Mapping of Cancer Risk. Cell 166, 1132-1146.e7 (2016).

19. Seitz, H. K. \& Stickel, F. Molecular mechanisms of alcohol-mediated carcinogenesis. Nat. Rev. Cancer 7, 599-612 (2007).

20. Desai, A., Sandhu, S., Lai, J.-P. \& Sandhu, D. S. Hepatocellular carcinoma in noncirrhotic liver: A comprehensive review. World Journal of Hepatology 11, 1-18 (2019).

21. Zhu, M. et al. Somatic Mutations Increase Hepatic Clonal Fitness and Regeneration in Chronic Liver Disease. Cell (2019). doi:10.1016/j.cell.2019.03.026

22. Khosla, R. et al. EpCAM+ Liver Cancer Stem-Like Cells Exhibiting Autocrine Wnt Signaling Potentially Originate in Cirrhotic Patients. Stem Cells Transl. Med. 6, 807818 (2017).

23. Chen, D. et al. Epithelial to mesenchymal transition is involved in ethanol promoted hepatocellular carcinoma cells metastasis and stemness. Mol. Carcinog. 57, 1358-1370 (2018).

24. Blokzijl, F. et al. Tissue-specific mutation accumulation in human adult stem cells 
during life. Nature 538, 260-264 (2016).

25. Jager, M. et al. Measuring mutation accumulation in single human adult stem cells by whole-genome sequencing of organoid cultures. Nat. Protoc. 13, 59-78 (2018).

26. Barker, N. et al. Crypt stem cells as the cells-of-origin of intestinal cancer. Nature 457, 608-611 (2009).

27. Adams, P. D., Jasper, H. \& Rudolph, K. L. Aging-Induced Stem Cell Mutations as Drivers for Disease and Cancer. Cell Stem Cell 16, 601-612 (2015).

28. Lee, J.-S. et al. A novel prognostic subtype of human hepatocellular carcinoma derived from hepatic progenitor cells. Nat. Med. 12, 410-416 (2006).

29. Tummala, K. S. et al. Hepatocellular Carcinomas Originate Predominantly from Hepatocytes and Benign Lesions from Hepatic Progenitor Cells. Cell Rep. 19, 584-600 (2017).

30. Holczbauer, Á. et al. Modeling pathogenesis of primary liver cancer in lineage-specific mouse cell types. Gastroenterology 145, 221-231 (2013).

31. $\mathrm{Mu}, \mathrm{X}$. et al. Hepatocellular carcinoma originates from hepatocytes and not from the progenitor/biliary compartment. Journal of Clinical Investigation 125, 3891-3903 (2015).

32. Alexandrov, L. B. et al. Signatures of mutational processes in human cancer. Nature 500, 415-421 (2013).

33. Nik-Zainal, S. et al. Landscape of somatic mutations in 560 breast cancer whole-genome sequences. Nature 534, 47-54 (2016).

34. Alexandrov, L. B. et al. The Repertoire of Mutational Signatures in Human Cancer. bioRxiv 322859 (2018). doi:10.1101/322859

542 35. Zou, X. et al. Validating the concept of mutational signatures with isogenic cell models. Nat. Commun. 9, 1744 (2018). 
36. Yokoyama, A. et al. Age-related remodelling of oesophageal epithelia by mutated cancer drivers. Nature 565, 312-317 (2019).

37. Supek, F. \& Lehner, B. Clustered Mutation Signatures Reveal that Error-Prone DNA Repair Targets Mutations to Active Genes. Cell 170, 534-547.e23 (2017).

38. Barski, A. et al. High-resolution profiling of histone methylations in the human genome. Cell 129, 823-837 (2007).

39. Bannister, A. J. et al. Spatial distribution of di- and tri-methyl lysine 36 of histone $\mathrm{H} 3$ at active genes. J. Biol. Chem. 280, 17732-17736 (2005).

40. Xu, Y., Tan, L.-J., Grachtchouk, V., Voorhees, J. J. \& Fisher, G. J. Receptor-type protein-tyrosine phosphatase-kappa regulates epidermal growth factor receptor function. J. Biol. Chem. 280, 42694-42700 (2005).

41. Luedde, T., Kaplowitz, N. \& Schwabe, R. F. Cell death and cell death responses in liver disease: mechanisms and clinical relevance. Gastroenterology 147, 765-783.e4 (2014).

42. Lu, W.-Y. et al. Hepatic progenitor cells of biliary origin with liver repopulation capacity. Nat. Cell Biol. 17, 971-983 (2015).

43. Sun, P.-H., Ye, L., Mason, M. D. \& Jiang, W. G. Protein tyrosine phosphatase kappa (PTPRK) is a negative regulator of adhesion and invasion of breast cancer cells, and associates with poor prognosis of breast cancer. J. Cancer Res. Clin. Oncol. 139, 1129-

44. Flavell, J. R. et al. Down-regulation of the TGF-beta target gene, PTPRK, by the Epstein-Barr virus encoded EBNA1 contributes to the growth and survival of Hodgkin lymphoma cells. Blood 111, 292-301 (2008). Hepatocellular Carcinoma: A Meta-Analysis. PLoS One 7, e32159 (2012). 
of hepatocellular carcinoma in patients with cirrhosis. JAMA 299, 53-60 (2008).

570

571

572

573

574

575

576

577

578

579

580

581

582

583

584

585

586

587

588

589

590

591

592

593

47. Sanchez-Vega, F. et al. Oncogenic Signaling Pathways in The Cancer Genome Atlas. Cell 173, 321-337.e10 (2018).

48. Schiffer, E. et al. Gefitinib, an EGFR inhibitor, prevents hepatocellular carcinoma development in the rat liver with cirrhosis. Hepatology 41, 307-314 (2005).

49. Mantovani, A., Allavena, P., Sica, A. \& Balkwill, F. Cancer-related inflammation. Nature 454, 436-444 (2008).

50. Shimizu, T., Marusawa, H., Endo, Y. \& Chiba, T. Inflammation-mediated genomic instability: roles of activation-induced cytidine deaminase in carcinogenesis. Cancer Sci. 103, 1201-1206 (2012).

51. Broutier, L. et al. Culture and establishment of self-renewing human and mouse adult liver and pancreas 3D organoids and their genetic manipulation. Nat. Protoc. 11, 17241743 (2016).

52. Huch, M. et al. Long-term culture of genome-stable bipotent stem cells from adult human liver. Cell 160, 299-312 (2015).

53. Li, H. \& Durbin, R. Fast and accurate short read alignment with Burrows-Wheeler transform. Bioinformatics 25, 1754-1760 (2009).

54. Boeva, V. et al. Control-FREEC: a tool for assessing copy number and allelic content using next-generation sequencing data. Bioinformatics 28, 423-425 (2012).

55. Chen, X. et al. Manta: rapid detection of structural variants and indels for germline and cancer sequencing applications. Bioinformatics 32, 1220-1222 (2016).

56. Quinlan, A. R. BEDTools: The Swiss-Army Tool for Genome Feature Analysis. Curr. Protoc. Bioinformatics 47, 11.12.1-34 (2014).

57. McKenna, A. et al. The Genome Analysis Toolkit: a MapReduce framework for analyzing next-generation DNA sequencing data. Genome Res. 20, 1297-1303 (2010). 
594

595

596

597

598

599

600

601

602

603

604

605

606

607

608

609

610

611

612

613

614

615

616

617

618

58. Van der Auwera, G. A. et al. From FastQ data to high confidence variant calls: the Genome Analysis Toolkit best practices pipeline. Curr. Protoc. Bioinformatics 43, 11.10.1-33 (2013).

59. Durinck, S. et al. BioMart and Bioconductor: a powerful link between biological databases and microarray data analysis. Bioinformatics 21, 3439-3440 (2005).

60. Durinck, S., Spellman, P. T., Birney, E. \& Huber, W. Mapping identifiers for the integration of genomic datasets with the R/Bioconductor package biomaRt. Nat. Protoc.

4, 1184-1191 (2009).

61. Carlson, M. \& Maintainer, B. P. TxDb.Hsapiens.UCSC.hg19.knownGene: Annotation package for TxDb object(s). (2015).

62. Blokzijl, F., Janssen, R., van Boxtel, R. \& Cuppen, E. MutationalPatterns: comprehensive genome-wide analysis of mutational processes. Genome Med. 10, 33 (2018).

63. Zhang, Y. et al. Model-based analysis of ChIP-Seq (MACS). Genome Biol. 9, R137 (2008).

64. Dobin, A. et al. STAR: ultrafast universal RNA-seq aligner. Bioinformatics 29, 15-21 (2013).

\section{ACKNOWLEDGEMENTS}

The authors would like to thank the Utrecht Sequencing Facility and the UBEC for sequencing and for input on the bioinformatic analyses, respectively. The UBEC is subsidized by the University Medical Center Utrecht and the Utrecht Sequencing Facility is subsidized by the University Medical Center Utrecht, Hubrecht Institute, and Utrecht University. This study was financially supported by the research program InnoSysTox (project number 114027003), by the Netherlands Organisation for Health Research and Development (ZonMw), by the Dutch 
619 Cancer Society (project number 10496) and is part of the Oncode Institute, which is partly

620 financed by the Dutch Cancer Society and was funded by the gravitation program

621 CancerGenomiCs.nl from the Netherlands Organisation for Scientific Research (NWO). We

622 thank the Hartwig Medical Foundation (Amsterdam, The Netherlands) for generating, 623 analyzing and providing access to reference whole genome sequencing data of the Netherlands 624 population.

625

626

\section{AUTHOR CONTRIBUTIONS}

627

R.L., J.J., J.I., M.D., and M.V. collected liver biopsies. M.J., E.K., and N.B. performed

628 organoid culturing. N.B. isolated the RNA and protein, prepared RNA-seq libraries of the

629 organoid cultures and performed Western blot. M.J., M.L., B.R., R.J., and S.B. performed bioinformatic analyses. M.J., E.K., M.V., R.B., L.L., and E.C. were involved in the conceptual design of this study. M.J. and E.C. wrote the manuscript. All authors provided textual comments and have approved the manuscript. R.B., L.L., and E.C. supervised this study.

633

634 AUTHOR INFORMATION

\section{Competing interests}

636 The authors declare no competing interests.

\section{Corresponding authors}

639 Correspondence to Edwin Cuppen. 
TABLES

\begin{tabular}{lllllll} 
& Entrez & & & & $q$ & $q$ \\
Gene & gene ID & Sample type & Sample(s) & Mutation type & (missense) & (nonsense) \\
\hline ATP1A1 & 476 & Healthy liver & $15-a$ & missense & 1.00 & 1.00 \\
PAX7 & 5081 & Healthy liver & $21-\mathrm{a}$ & missense & 1.00 & 1.00 \\
PREX2 & 80243 & Healthy liver & $21-\mathrm{b}$ & missense & 1.00 & 1.00 \\
\hdashline PTPRK & 5796 & Alcoholic liver & alc3-a, alc5-a & nonsense, nonsense & 1.00 & 0.02 \\
ALK & 238 & Alcoholic liver & alc3-b & missense & 1.00 & 1.00 \\
CACNA1D & 776 & Alcoholic liver & alc3-b & missense & 1.00 & 1.00 \\
ZNF331 & 55422 & Alcoholic liver & alc4-b & missense & 1.00 & 1.00 \\
CUX1 & 1523 & Alcoholic liver & alc5-a & missense & 1.00 & 1.00 \\
TERT & 7015 & Alcoholic liver & alc5-a & missense & 1.00 & 1.00 \\
\hdashline CD274 & 29126 & HCC & HCC-trunk & missense & 1.00 & 1.00 \\
CIITA & 4261 & HCC & HCC-trunk & missense & 1.00 & 1.00 \\
KLF4 & 9314 & HCC & HCC-trunk & missense & 1.00 & 1.00 \\
MUC1 & 4582 & HCC & HCC-trunk & missense & 1.00 & 1.00 \\
\hline
\end{tabular}

641

642 Table 1 Somatic missense and nonsense base substitutions in cancer genes observed in healthy liver

643 ASCs, alcoholic liver ASCs, and the MRCA of an HCC. $q$ values (likelihood ratio test, FDR correction)

644 indicate significant enrichment of nonsynonymous base substitutions within genes. 
FIGURES

a

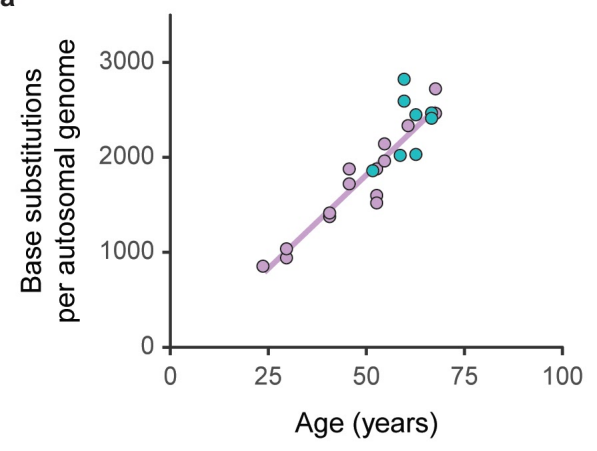

-o- Healthy liver $\bigcirc$ Alcoholic liver b

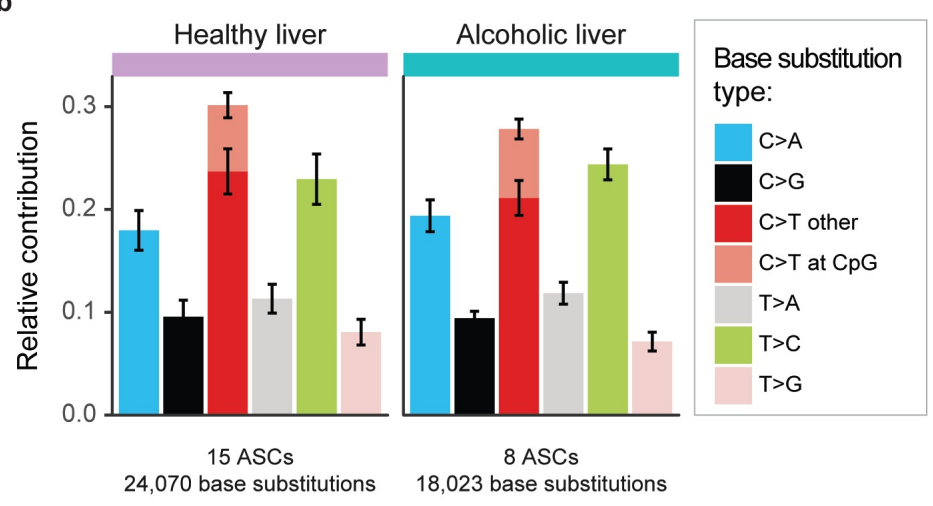

c

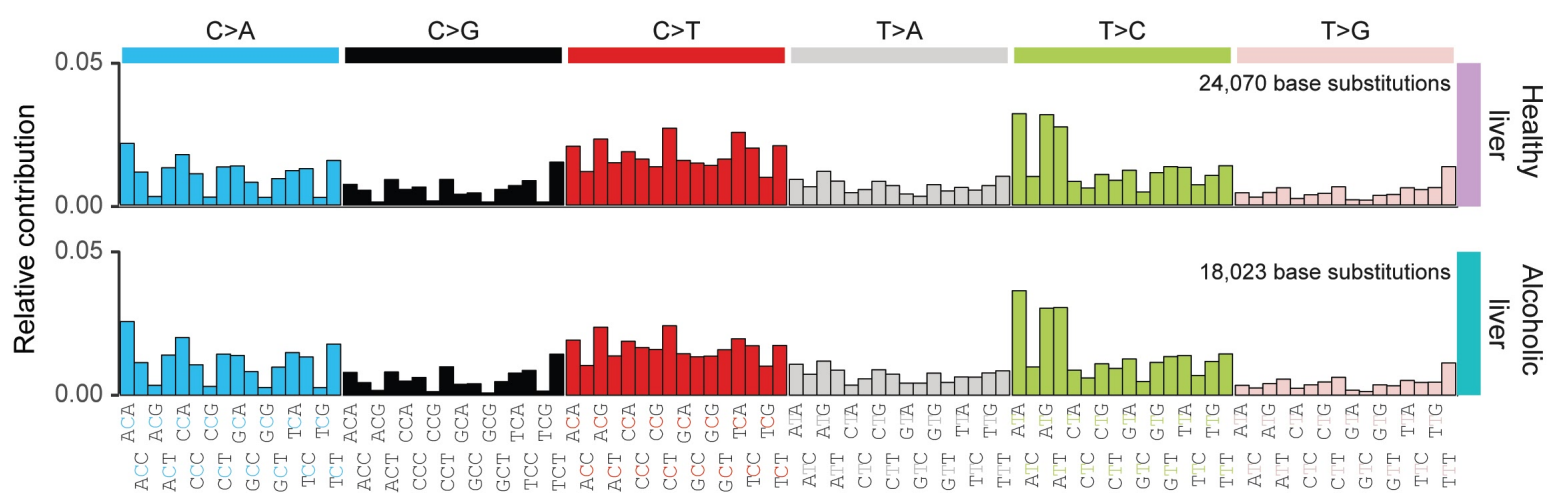

647 Fig. 1 Somatic base substitutions in healthy and alcoholic liver stem cells. a Number of somatic base

648 substitutions in the autosomal genomes of 15 healthy and 8 alcoholic liver stem cells of 9 and 5 donors,

649 respectively. Each stem cell is represented by a data point. A linear accumulation of base substitutions

650 with age was observed in healthy liver, indicated by the purple trendline. b Mean relative contribution

651 of the base substitution types to the mutation spectra of healthy and alcoholic liver ASCs. Error bars

652 represent standard deviation. c Mean relative contribution of 96 context-dependent base substitution types to the mutational profiles of healthy and alcoholic liver ASCs. 
a

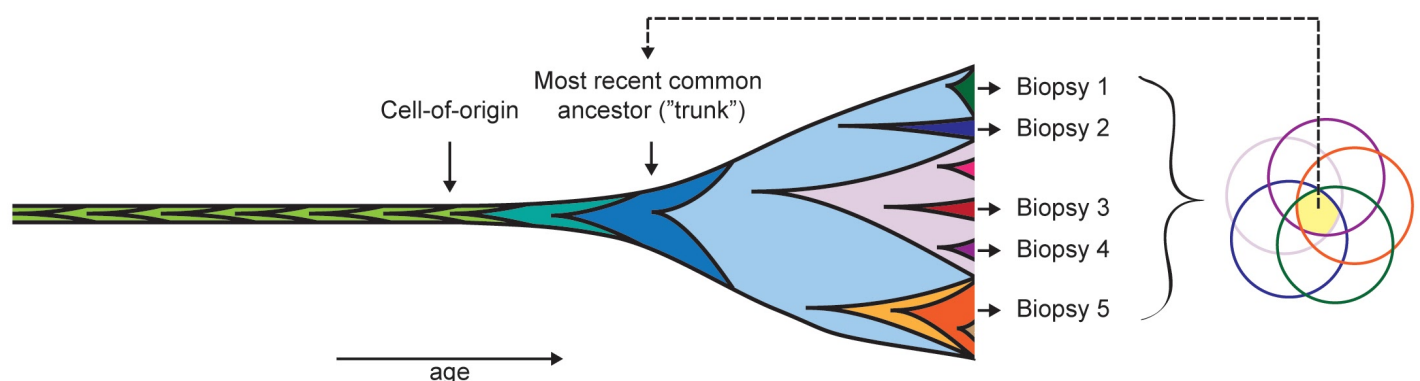

b
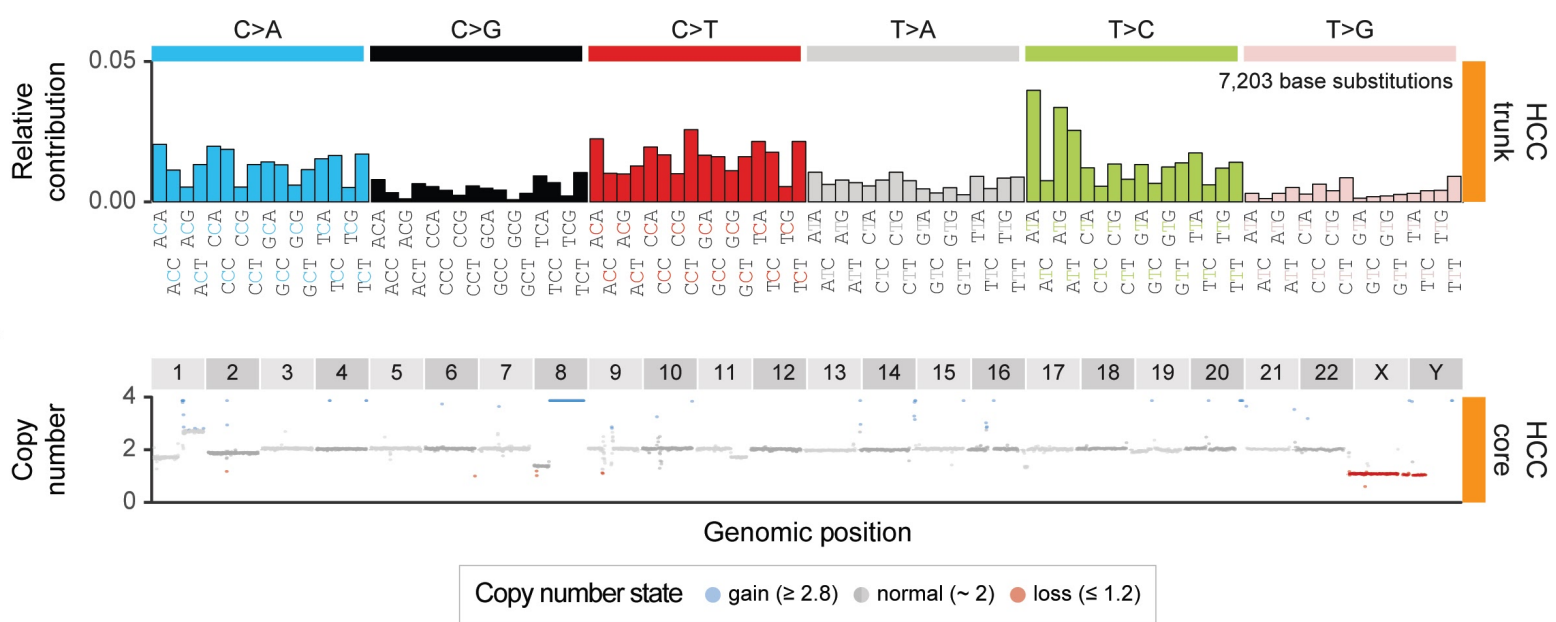

655 Fig. 2 Somatic mutations in the most recent common ancestor of an HCC in a patient with a history of

656 chronic alcohol intake. a Mutations that accumulated in the most recent common ancestor ("trunk") can

657 be identified, by determining the mutations that are shared by multiple biopsies (in yellow). Five

658 biopsies across a $13 \mathrm{~cm} \mathrm{HCC}$ were sequenced to identify the trunk mutations. b Relative contribution of

65996 context-dependent base substitution types to the mutational profile of the recent common ancestor

660 of an HCC. c Genomic copy number profile of one of the HCC biopsies (HCC-core), which is representative for all biopsies (Supplementary Fig. 4c). 
a

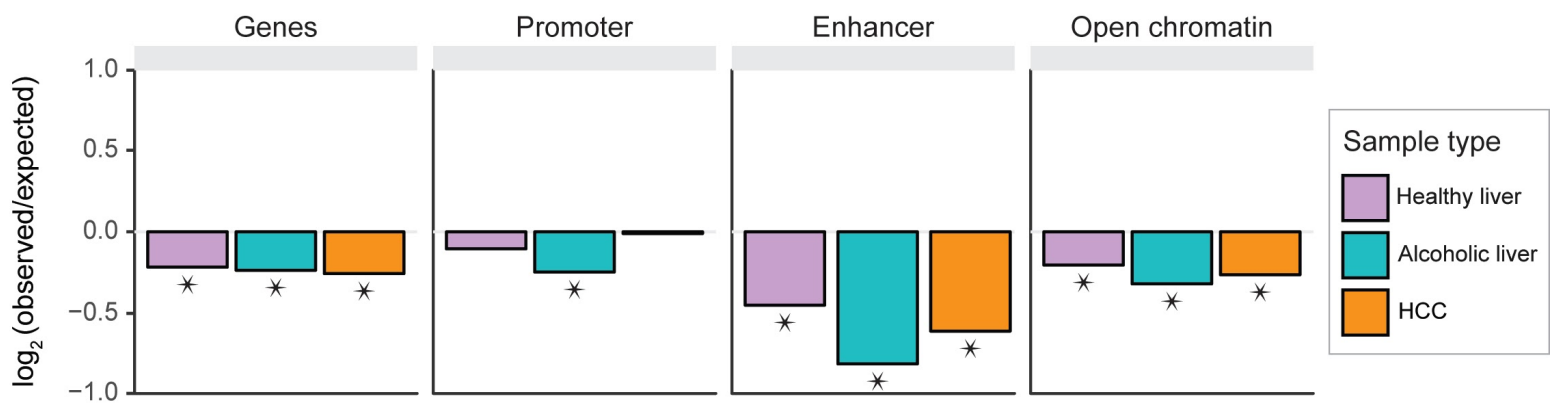

b

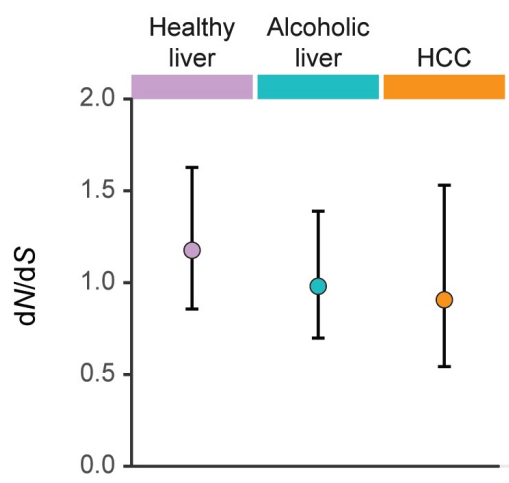

c

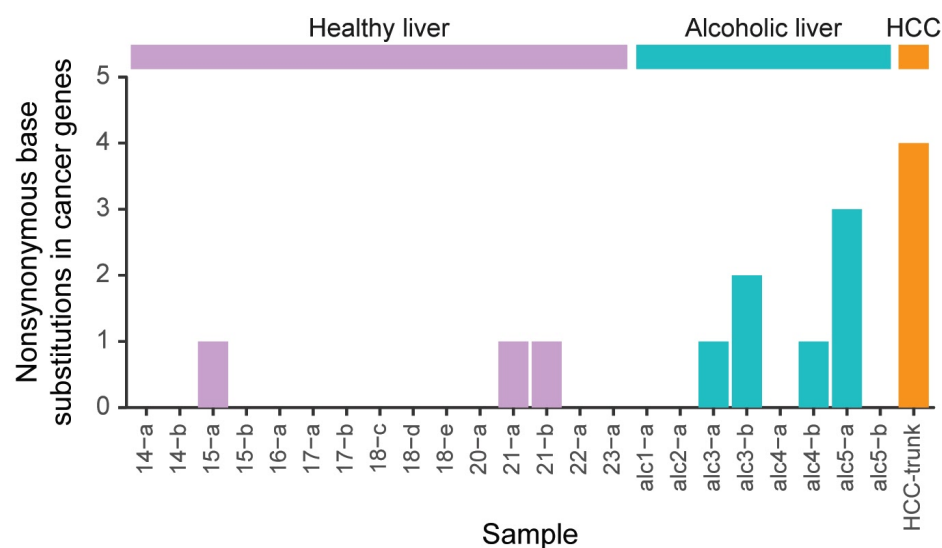

662

663 Fig. 3 Genomic distribution of the somatic base substitutions acquired in healthy liver stem cells,

664 alcoholic liver stem cells, and the most recent common ancestor of an HCC. a The effect size of the

665 depletion of somatic base substitutions in genes, promoters, enhancers, and open chromatin regions.

666 Asterisks indicate significant depletion. b $\mathrm{d} N / \mathrm{d} S$ of the somatic base substitutions in genes in the

667 indicated sample types. Data points represent the Maximum-likelihood estimates and error bars

668

669 represent the $95 \%$ confidence intervals. c Number of nonsynonymous base substitutions in cancer genes in each indicated sample. 


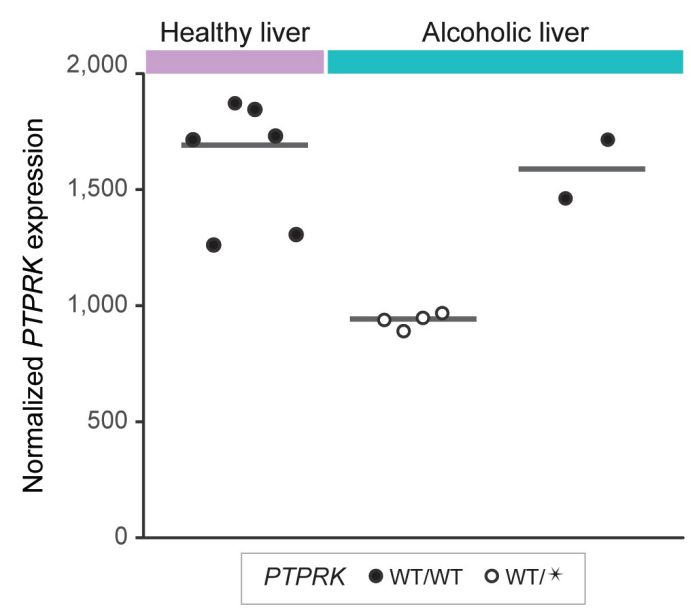

b

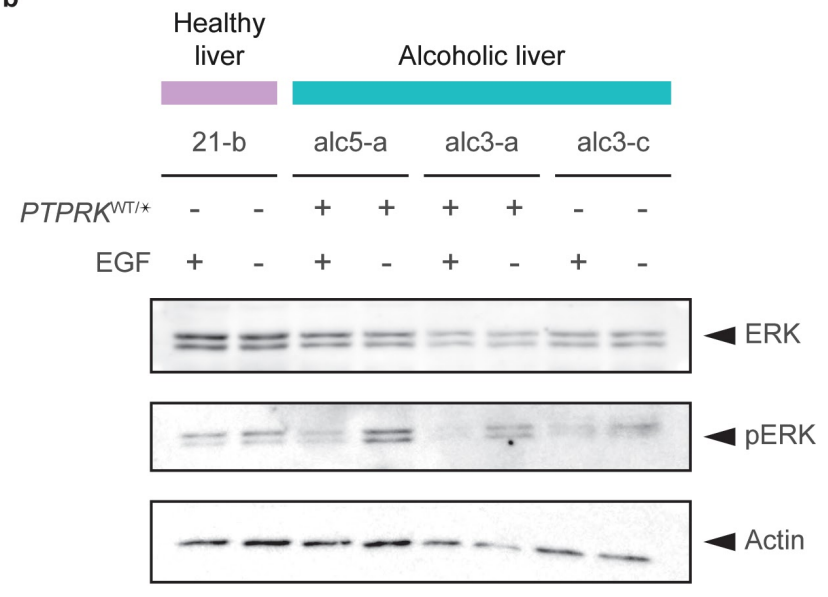

671 Fig. 4 Functional consequences of nonsense PTPRK base substitutions. a Normalized PTPRK mRNA

672 expression in alcoholic $P T P R K^{\mathrm{WT} / *}$, healthy $P T P R K^{\mathrm{WT} / \mathrm{WT}}$ and alcoholic $P T P R K^{\mathrm{WT} / \mathrm{WT}}$ liver organoid

673 cultures. Normalized counts were calculated for duplicate measures of 2 alcoholic PTPRK ${ }^{\mathrm{WT} / *}, 3$

674 healthy $P T P R K^{\mathrm{WT} / \mathrm{WT}}$, and 1 alcoholic $P T P R K^{\mathrm{WT} / \mathrm{WT}}$ organoid cultures. Each data point represents a

675 single measurement. Lines indicate median PTPRK expression per sample type. WT $=$ wildtype, $*=$

676 nonsense base substitution. b Western blot of ERK, pERK and actin in organoid cultures of PTPRK ${ }^{\mathrm{WT} / *}$

677 livers and healthy and alcoholic $P T P R K^{\mathrm{WT} / \mathrm{WT}}$ liver cultures with and without EGF in the culturing 678 medium. 